\title{
Análisis de las necesidades del cliente y su satisfacción en la industria del mueble según las normas ISO 9000: un estudio de casos
}

\author{
Analysing customer requirements and satisfaction \\ in the furniture industry according to ISO 9000 \\ certification: a case study
}

\section{Laura Martínez Caro / Enrique Flores López / Jose Antonio Martínez García ${ }^{1}$ \\ Universidad Politécnica de Cartagena}

Recibido el 1 de junio de 2009, aceptado el 25 de enero de 2010

$\mathrm{N}^{\mathrm{o}}$ de clasificación JEL: M1: Administración de Empresas, M3: Marketing y Publicidad

DOI: $10.5295 / \mathrm{cdg} .100162 \mathrm{~lm}$

Reseña Bibliográfica: MARTÍNEZ CARO, L.; FLORES LÓPEZ, E.; MARTÍNEZ GARCÍA J. A. (2010): "Análisis de las necesidades del cliente y su satisfacción en la industria del mueble según las normas ISO 9000: un estudio de casos"; Cuadernos de Gestión, Vol. 10. № 2, pp. 99-116. DOI: $10.5295 / \mathrm{cdg} .100162 \mathrm{~lm}$

\section{Resumen:}

La necesidad de determinar los requerimientos del cliente y satisfacerlos se ha convertido en el principal objetivo de las empresas. Gestionar esa actividad, comúnmente asociada al departamento de marketing, ha llegado a ser el fin de cualquier sistema de calidad, y por ende, del resto de departamentos de la organización. A través de un estudio de casos, este trabajo pretende identificar cuales son las prácticas de marketing habituales en la industria del mueble, y plantear un marco de actuación idóneo de acuerdo con los procedimientos establecidos en las normas de calidad ISO 9000:2000.

Palabras clave:

Necesidades del cliente, satisfacción del cliente, ISO 9000:2000.

\begin{abstract}
:
The need to identify customer requirements and to satisfy them has become the main objective of companies. To manage that activity, that it is commonly associated to the marketing department, is the aim of every quality system, and therefore is a task that comprises the remaining departments of the organization. Through a case study, this paper seeks to identify which are the usual marketing practices in the furniture industry, and to outline an appropriate performance framework in agreement with the procedures settled down in the ISO 9000:2000 standards.
\end{abstract}

Keywords:

Consumer needs, consumer satisfaction, ISO 9000:2000 certification.

${ }^{1}$ Universidad Politécnica de Cartagena. Departamento de Economía de la Empresa. Paseo Alfonso XIII, 50. C.P. 30203.e-mail: laura.martinez@upct.es; enrique.flores@upct.es; josean.martinez@upct.es 


\section{INTRODUCCIÓN}

Para una empresa el tener una calidad superior a la de su competencia le supone contar con un factor clave para generar ventajas competitivas. El valor estratégico que implica la ventaja en calidad radica en que ésta puede contribuir a multiplicar la sostenibilidad de las ventajas competitivas de la empresa al generar fidelidad del cliente a través de su satisfacción (Anderson et al., 1994). Asimismo, pueden "contribuir también a crear ventajas competitivas en costes, al enfocarse a la reducción de los gastos provocados por el despilfarro de recursos y los errores internos y externos a la organización" (Camisón, 1996, p. 105).

Por estos motivos, el interés por la calidad en las empresas es creciente, con independencia del sector de actividad que se tome de referencia, lo que ha llevado a que se establezcan progresivamente una serie de estándares y normas de calidad, como las normas ISO 9000 (International Organization for Standardization) o el modelo EFQM, que afectan tanto a la organización de las actividades como a la configuración de los propios productos (Hernández et al., 2003). Este importante desarrollo de los estándares de actuación es fruto tanto del interés de las autoridades públicas, quienes potencian la calidad como estrategia empresarial, como de los propios clientes, que son cada vez más exigentes y reflejan sus preferencias en el proceso de evaluación y selección de las empresas (Hernández et al., 2003).

En este sentido, uno de los acontecimientos más importantes que han tenido lugar en el ámbito de la calidad en las tres últimas décadas ha sido la aparición de las normas internacionales ISO 9000, que por su carácter global homogenizan los procesos de mejora de la calidad en las empresas de todo el mundo (Escanciano, 2001).

La mayoría de los estudios efectuados sobre la certificación ISO 9000 recaban la opinión de las empresas acerca de su eficacia (Escanciano, 2001) en el ámbito de gestión, pero poco se ha investigado sobre la repercusión en términos de marketing que ésta certificación tiene. La norma recoge, en varios de sus apartados, una serie de consideraciones y aspectos que inciden de lleno en el ámbito del marketing de las empresas, al establecer la satisfacción del cliente como uno de los principios básicos de la norma ${ }^{1}$. Sin embargo, ya que la norma es bastante genérica (es aplicable a las compañías manufactureras y de servicios de todos los sectores) no permite crear un modelo de implantación para todo tipo de compañías. Por ello, el objetivo de esta investigación cualitativa es analizar en un tejido empresarial concreto, la industria del mueble, si las empresas están llevando a cabo correctamente todos aquellos postulados relacionados con la identificación de las necesidades del consumidor y su satisfacción propuestos por las normas ISO 9000, y que pueden tener una importante incidencia en el logro de la calidad global de una organización. Por medio de la metodología del estudio de casos, podemos tener una representación de cómo se está

\footnotetext{
${ }^{1}$ En el Apartado 1.1. Generalidades. Establece que "Esta norma internacional especifica los requisitos para un sistema de gestión de la calidad, cuando una organización: a) necesita demostrar su capacidad para proporcionar de forma coherente productos que satisfagan los requisitos del cliente y los reglamentarios aplicables y b) aspira a aumentar la satisfacción del cliente a través de la aplicación eficaz del sistema, incluidos los procesos para la mejora continua del sistema y el aseguramiento de la conformidad con los requisitos del cliente y los reglamentarios aplicable."
} 
actuando realmente en este contexto específico y proponer actuaciones concretas en el ámbito comercial para aplicar de la forma más eficiente las ideas contenidas en la norma y que apenas están detalladas en ésta.

\section{MARCO CONCEPTUAL}

En los últimos años, la industria del mueble se ha consolidado como uno de los principales motores de la economía nacional. Sin embargo, el sector tiene sobre sí una peligrosa amenaza: la importación. El mueble español está sufriendo desde hace unos años una fuerte crisis, provocada sobre todo por la pérdida de competitividad de sus empresas, respecto a un mercado extranjero con el que, en la mayoría de ocasiones, no se puede competir en precios. La Asociación Nacional de Industriales y Exportadores de Muebles de España (ANIEME) ubica los principales mercados de origen de muebles importados a España en China, principal país importador, con un volumen de negocio de 245 millones de euros. Al gigante asiático le siguen Italia, con 176, y Alemania, con 143 millones de euros (datos referentes al año 2006).

Dada la imposibilidad de competir en precio con las economías emergentes, el mercado español debe buscar otra estrategia de diferenciación, y la calidad es una de ellas, innovando en aspectos como la atención al cliente, proponiendo un servicio exclusivo y una garantía de marca (Sastre, 2004).

Tradicionalmente la industria del mueble ha sido considerada como un negocio local, donde la PYME mantiene un papel predominante. Este tipo de empresas, prefieren implantar sistemas de calidad ISO 9000 antes que adoptar otros enfoques como la Gestión de la Calidad Total (Urbonavicius, 2005). Esto es debido a que las PYMES tienen menos recursos financieros para invertir en prácticas de gestión de la calidad y consecuentemente deben elegir aquella alternativa que le puede proporcionar los resultados más rápidos al menor coste.

Las normas ISO 9000 son una herramienta de gestión que tiene como base la sistematización y formalización de tareas para lograr una uniformidad en el producto o servicio y la conformidad en el cumplimiento de las especificaciones establecidas por el cliente (Anderson et al, 1999). El principal objetivo de las normas ISO 9000:2000 es relacionar la gestión moderna de la calidad con los procesos y actividades de una organización, incluyendo la promoción de la mejora continua y el logro de la satisfacción del cliente (AENOR, 2001).

En esta declaración de principios las normas incluyen dos variables muy interrelacionadas: calidad y satisfacción. Las normas ISO 9000 (AENOR, 2001) ofrecen una definición de calidad bastante genérica y desde un punto de vista objetivo entiende la calidad como "el grado en el que un conjunto de características inherentes cumple con los requisitos". Por su parte, la satisfacción puede ser entendida como una respuesta emocional, que resulta del proceso cognitivo de evaluación del bien/servicio recibido frente a los costes del obtenerlo (Rust y Oliver, 1994).

La satisfacción se ha convertido en un aspecto extremadamente importante para el éxito de algunos negocios debido al entorno tan dinámico y la intensiva competitividad de su mercado. Por ello, si no se reconoce y responde rápida y eficientemente a las ne- 
cesidades de los consumidores, una empresa podría aumentar su carga de trabajo, perder oportunidades de negocio, y en última instancia, incrementar el nivel de insatisfacción de sus consumidores (Gurau y Ranchhod, 2002).

En muchas organizaciones el interés por la satisfacción del cliente nace como fruto de la implantación de programas de calidad total (Mohr-Jackson, 1998). En esta misma línea, Rodríguez y González (2003) señalan que cuando una organización decide certificarse es cuando desea aumentar la satisfacción del cliente, proporcionando bienes o servicios que cumplan los requisitos de dichos clientes y las reglamentaciones exigidas.

A lo largo de las normas ISO 9000:2000 son muchos los aspectos que inciden en la conveniencia de determinar las necesidades del cliente y su satisfacción. Para cumplir este aspecto, se requiere que la organización tenga un enfoque al cliente y haga un seguimiento de la satisfacción del mismo. Dichas exigencias entran dentro de las competencias atribuidas al marketing y en muchos casos pasan desapercibidas por los directores administrativos más preocupados por los aspectos productivos y de costes.

Dada su amplia relevancia en la gestión empresarial, la certificación ISO 9000 ha sido objeto de abundantes investigaciones; el fin perseguido por éstas puede agruparse del siguiente modo:

1) Determinar la incidencia de la gestión de la calidad en la mejora de bienes y servicios (ej. Phillips, et al., 1983; Rust et al., 1994; Brown y van der Wiele, 1995).

2) Estudiar los resultados financieros debido a la implantación de la norma (ej. Flynn et al., 1997; Caruana y Pitt, 1997; Chapman et al., 1997; Chow-Chua et al., 2003; Dimara et al., 2004).

3) Analizar la relación de las normas ISO 9000 con la Gestión de Calidad Total (ej. Escanciano et al., 2001; Gotzamani y Tsiotras, 2002; Magd y Curry, 2003b; Martínez y Martínez, 2004; Casadesús et al., 2004).

4) Analizar la motivación para la implantación de la normativa y los resultados que aporta ésta (ej. Carlsson y Carlsson, 1996; Buttle, 1997; Lee et al., 1999; Casadesús y Giménez, 2000; Heras et al., 2000; Santos y Escanciano, 2000; Marimon et al., 2002; Álvarez Gil et al., 2002; Calisir et al., 2005; Zaramdini, 2007).

A pesar de las numerosas investigaciones relacionadas con las normas que se han realizado, son escasas las investigaciones que analizan explícitamente las prácticas de marketing asociadas a ellas. Tan sólo el estudio de Fernández y Prado (2005) analiza las prácticas de medición y análisis de la satisfacción del cliente empleadas por empresas certificadas de Galicia y norte de Portugal. Sus resultados muestran cierto abuso de la encuesta postal, en la que detectan algunas deficiencias metodológicas respecto a su contenido. No obstante, dentro de los estudios que examinan los resultados que aportan las normas, muchos de ellos analizan la implicación de la certificación en la satisfacción de consumidor (Brown y van de Wiele, 1995; Terziovski et al., 1995; Tsiotras y Gotzamani, 1996; Buttle, 1997; Casadesús et al., 2004; Rodríguez y González, 2003; Heras et al., 2005; Marín et al., 2007; Calisir, 2007; Martinez y Martinez, 2009) y en la identificación de las necesidades o requerimientos del cliente (Quazi y Padibjo, 1997; Jones et al., 1997; Brown et al., 1998; Casadesús y Giménez, 2000; Magd y Curry, 2003a). Sin embargo, ninguno de estos estudios identifica cuales son las prácticas de marketing más adecuadas para alcanzar los requisitos impuestos en las normas, tan sólo analizan en que medida la satisfacción ha sido mejorada o no por hecho de implantar un sistema de calidad. 
Ante la falta de manuales que indiquen u orienten en la detección y satisfacción de las necesidades del cliente, este estudio analiza como las empresas del sector del mueble llevan a cabo estas actuaciones, es decir, ¿están las empresas haciendo todo lo posible por satisfacer a sus clientes? ¿están aplicando las mejores técnicas para conocer sus niveles de satisfacción? ¿cómo determinan las necesidades de sus clientes?. Las respuestas a estas y otras muchas preguntas nos permitirán definir globalmente la actuación de esta industria en los aspectos relacionados con el marketing para la consecución de la mejora en la calidad y, por ende, de la orientación al consumidor.

\section{METODOLOGÍA}

La metodología empleada para el analizar la actuación de las empresas del mueble en relación al cliente fue el estudio de casos. Según Yin (2003) éste es el procedimiento más adecuado para entender un fenómeno en su contexto real. Por lo tanto, el estudio cualitativo es útil cuando: (1) el fenómeno bajo estudio es amplio y complejo; (2) cuando el conocimiento sobre el tema es insuficiente para permitir el empleo de cuestiones causales; (3) cuando es necesaria una investigación holística, en profundidad, y (4) cuando un fenómeno no puede ser estudiado fuera del contexto donde ocurre (Bonoma, 1985; Benbasat et al., 1987; Yin, 1994). De este modo, esta metodología permite identificar temas comunes y presentes en la industria del mueble con la intención de identificar oportunidades en la mejora de su calidad.

El estudio de casos intenta conseguir un conocimiento extensivo sobre un caso único o sobre un pequeño numero de casos. El estudio de un caso único investiga en profundidad sobre una entidad, una compañía, una decisión o una región. En el estudio de múltiples casos, dos o más entidades son estudiadas para dar la oportunidad de comparar, siendo esta alternativa deseable cuando el fin de la investigación es construir y testar una teoría (Yin, 2003). Por lo tanto, y teniendo en cuenta que el objetivo de nuestro estudio es analizar en que medida la industria del mueble está realizando todas las recomendaciones recogidas en las normas ISO para detectar y satisfacer las necesidades del consumidor, realizamos un estudio de casos múltiple.

El número de empresas del mueble estudiadas (casos) fueron 27, todas ellas con sede en la Región de Murcia, uno de los núcleos principales donde se concentran las empresas del sector mobiliario. Estas compañías fueron seleccionadas de la base de datos SABI (Sistema de Análisis de Balances Ibéricos). Esta base contiene información financiera sobre más de 500.000 empresas españolas y portuguesas.

El medio empleado para obtener la información acerca de los procedimientos de calidad empleados fue la entrevista personal. Este método permite a los investigadores abordar cuestiones complejas y explicar posibles malentendidos. De acuerdo con Yin (2003) las entrevistas son una manera flexible de recoger los datos y centrarse directamente en el objetivo de estudio. Las entrevistas fueron la principal fuente de cada caso, complementadas por la documentación económica y financiera procedente del SABI.

Los datos fueron recogidos durante Septiembre y Diciembre de 2005 por medio de entrevistadores. Las entrevistas fueron realizadas a los directivos de calidad y duraron generalmente una hora, hora y media. El 96,30\% de las empresas encuestadas eras PYMEs y 
el 40,74\% estaban certificadas con la ISO 9000. El producto principal que comercian esas empresas puede contemplarse en la Tabla 1.

Tabla 1

Producto principal de las empresas entrevistadas

\begin{tabular}{l|c}
\hline \multicolumn{1}{c|}{ Producto principal } & $\mathrm{N}$ \\
\hline Modulares, comedores y dormitorios & 8 \\
\hline Fabricación de mueble tapizado & 5 \\
\hline Tapizados & 3 \\
\hline Fabricación de sillas & 2 \\
\hline Mueble auxiliar & 2 \\
\hline Mueble de cocina & 2 \\
\hline Mueble juvenil & 2 \\
\hline Componentes para mesas & 1 \\
\hline Mueble contemporáneo y moderno & 1 \\
\hline Sofás y mesas & 1 \\
\hline TOTAL & 27 \\
\hline
\end{tabular}

En aras de incrementar la validez de la investigación, en las entrevistas se empleo un cuestionario semi-estructurado. Éste fue diseñado teniendo en cuenta la literatura actual sobre calidad, con el fin de responder a los problemas y cuestiones de investigación. El cuestionario empleado fue leído y comentado por varios investigadores con la finalidad de comprobar la comprensión de las cuestiones y mejorar su formulación. El cuestionario estaba dividido en varias secciones: cuestiones generales (número de empleados, producto principal, posesión de la certificación) comunicación (medios que utilizan, comunicación con los empleados, proveedores, clientes, etc.) y acciones de marketing (tipo de vendedores, sistema de reclamaciones, prueba de mercado, asistencia a ferias, evaluación de los competidores, objetivos comerciales, diseño, satisfacción clientes, imagen).

Las entrevistas a cada empresa fueron realizadas por al menos dos investigadores, y éstos tomaron notas detalladas de forma individual, que fueron posteriormente puestas en común. Asimismo, las entrevistas fueron grabadas, lo que permitió a los investigadores contar con otro recurso para comprobar que no habían perdido información relevante. Además, durante el proceso de evaluación y manipulación de los datos los investigadores trataron de ser lo más objetivos posible para asegurar la fiabilidad de la investigación.

\section{RESULTADOS}

Tal y como se expuso anteriormente, la norma recoge, en varios de sus apartados, una serie de consideraciones y aspectos que inciden de lleno en el ámbito del marketing de 
las empresas, otorgándole, de este modo, una finalidad a todo el proceso de gestión de la calidad: la satisfacción del cliente. Así, en la Introducción de la norma ISO 9001, en el epígrafe 0.2 , se señala que la norma "promueve la adopción de un enfoque basado en procesos cuando se desarrolla, implementa y mejora la eficacia de un sistema de gestión de la calidad, para aumentar la satisfacción del cliente mediante el cumplimiento de sus requisitos", y en el apartado 5.2 de dicha norma se alude a que es responsabilidad de la Dirección el enfoque al cliente como una forma de aumentar la satisfacción del mismo, lo que supone una cuestión trascendental clave para el futuro de la organización, a este propósito de identificación y satisfacción de necesidades.

Por ello, hemos tratado de analizar aquellos aspectos relacionados con la identificación de las necesidades del consumidor y su satisfacción, que pueden tener una importante incidencia en el logro de la calidad global en una organización. Nuestros comentarios los basaremos en la norma ISO 9001; y en la norma ISO 9004, que establece directrices orientativas para la aplicación de la norma ISO 9001 "y para la mejora continua del desempeño" (tal y como indica la propia norma ISO 9001 en su epígrafe 0.3), y que en el ámbito comercial es muy esclarecedora, proponiendo actuaciones específicas que desarrollan detalladamente las ideas contenidas en la norma ISO 9001.

Como resultado del análisis crítico de las entrevistas en profundidad realizadas, tanto a empresas certificadas como no certificadas ${ }^{2}$, se ha podido observar una serie de deficiencias a la hora de cumplir con el objetivo de conocer y satisfacer las necesidades del cliente, que para un mejor análisis hemos agrupado, atendiendo a su naturaleza, en dos grandes bloques: (1) Deficiencias relativas a la detección de necesidades; y (2) Deficiencias relativas a la satisfacción de dichas necesidades.

\section{A) Deficiencias relativas a la detección de necesidades}

Varias de las deficiencias que hemos apreciado en el trabajo de campo, limitan la posibilidad de que las empresas puedan detectar las necesidades, preferencias y gustos de los consumidores, lo que obstaculizaría la consecución de altos niveles de calidad total en dichas organizaciones, por la enorme trascendencia que este conocimiento tiene en el establecimiento de un sistema de gestión de la calidad basado en procesos, tal y como claramente señala la normativa ISO 9001en su Introducción, en el epígrafe 0.2. En el ámbito de la satisfacción de necesidades, proceder con un enfoque basado en procesos, implica que la Dirección reconozca la existencia de muchas fuentes de información relativas al cliente, y establezca procesos eficaces y eficientes para recopilar, analizar y utilizar esta información para mejorar el desempeño de la organización. Sin embargo, en este ámbito, lo que hemos observado en numerosas empresas, mediante las entrevistas que hemos realizado, es los siguiente:

\footnotetext{
${ }^{2}$ Se decidió que los casos estudiados fueran tanto empresas certificadas con la ISO 9000 como no certificadas. El hecho de no estar certificado no excluye a estas empresa de estar actuando correctamente en las cuestiones relacionadas con el cliente, y viceversa, es decir, el hecho de estar certificado no significa que se este actuando de la forma más optima posible. Un par de empresas nos indicaron que no aún la poseían pero tenían la intención de conseguirla en breve por lo que ya empleaban criterios de calidad en su gestión.
} 
- Falta de un proceso formal de análisis de tendencias y preferencias del mercado. Muchas empresas viajan, más o menos ocasionalmente, a ferias y exposiciones, y en sus decisiones cuenta mucho la intuición personal del responsable o un somero análisis de las ventas pasadas propias o de la competencia. Pero con estas actuaciones, difícilmente, se podrán identificar y satisfacer las necesidades, requisitos y expectativas de los clientes, tal y como demanda la propia norma ISO 9001. En su epígrafe 8.4. la norma vincula el análisis de datos a "la satisfacción del cliente" entre otros fines, lo que configura un enfoque para la toma de decisiones basado en hechos, más que en opiniones subjetivas o intuiciones del decisor. En cuanto al análisis de ventas pasadas propias o ajenas, no deja de ser una fuente de información insuficiente, pues difícilmente puede detectar nuevas tendencias o cambios en los gustos, preferencias o necesidades de los consumidores.

- Falta de un proceso formal y estandarizado de análisis de la competencia. En algunas de las empresas analizadas, ocasionalmente se analizaban algunos productos (fundamentalmente novedades) de la competencia ${ }^{3}$, pero el proceso de evaluación seguido distaba mucho de ser sistemático, estandarizado y exhaustivo, como debería serlo. Este aspecto es importante porque, aunque la norma 9001 no lo cita expresamente, el análisis sistemático de las acciones de los competidores es una fuente útil de evaluación de las necesidades del mercado, tal y como la norma ISO 9004 propone en su epígrafe 8.2.1.2, equiparándolos a las encuestas dirigidas a clientes y usuarios. Además, si tenemos en cuenta que la misma norma ISO 9004, en su epígrafe 5.4.1, incluye el análisis comparativo y de los competidores como un medio adecuado para poder establecer los objetivos de calidad de la empresa, concluiremos en la necesidad de que las empresas del sector mejoren en este desempeño.

- Ausencia, casi generalizada, de prácticas formales de investigación de mercados como podría ser el testado de nuevos productos. El testado de productos podría ser un instrumento útil y de escaso coste para comprobar si los productos fabricados se corresponden, realmente, con la satisfacción de necesidades y/o la adecuación a los requisitos de los clientes; sin embargo su uso es prácticamente nulo. Esta ausencia de prácticas formales que investiguen el mercado contradicen lo que la propia norma ISO 9001 establece, en su epígrafe 8.2.1, cuando afirma que deben determinarse en la organización diferentes métodos que permitan percibir la satisfacción del cliente. En este mismo sentido, la norma ISO 9004 en su epígrafe 8.2.3 contempla la posibilidad de que se elaboren ensayos y pruebas para "asegurar que los productos son conformes con los requisitos y cuando se consideren las necesidades y expectativas del cliente (...)", y en el epígrafe 7.1.3.3 manifiesta que la Dirección debe asegurarse "que la validación de los productos demuestre que éstos cumplen las necesidades y expectativas de los clientes y otras partes interesadas. Las actividades de validación incluyen modelado, simulación y ensayos/pruebas, así como revisiones que involucren a los clientes o a otras partes interesadas".

\footnotetext{
${ }^{3}$ Prácticamente la totalidad de empresas analizadas, con o sin certificación, reconocen que su seguimiento de la competencia se limita a lo ven en la Ferias. Incluso, alguna empresa reconoce que los propios clientes son quienes les informan del estado de la competencia.
} 
- Falta de comunicación directa de las empresas con sus clientes (los establecimientos de venta), y con los usuarios finales de sus productos (los consumidores). La norma ISO 9001 establece en su apartado 7.2 (Procesos relacionados con el cliente) que la organización debe determinar y revisar los requisitos del producto especificados por el cliente. Y además en su apartado 7.2.3, establece que "la organización debe determinar e implementar disposiciones eficaces para la comunicación con los clientes”. Claramente, las empresas del sector del mueble fallan en el cumplimiento de este último apartado; y además, la información que les llega del mercado está sesgada por los intereses particulares de los comisionistas, o se limita a la recepción de quejas. Por un lado, la mayoría de las empresas del sector están indefensas ante sus propios intermediarios, que suelen favorecer a quienes mejores comisiones les ofrecen, al tiempo que la gran mayoría no puede contar con una red de vendedores propios, o aumentar sustancialmente las comisiones establecidas con sus comisionistas actuales. Por lo tanto, la información procedente de éstos puede ser interesada y no ser veraz. Por otro lado, el análisis de quejas puede ser un referente para retroalimentar las necesidades de los clientes, tal y como la norma recoge. El epígrafe 5.6.2 indica que "la retroalimentación del cliente" es una de las informaciones básicas con las que la Dirección debe contar para la revisión, y que los resultados de dicha revisión deben incluir "todas las decisiones y acciones relacionadas con la mejora del producto en relación con los requisitos del cliente" (apartado 5.6.3.b). Sin embargo, el análisis de quejas es un dato insuficiente, porque además de basarse en un hecho puntual (un cliente puede quejarse del retraso en la entrega de un sillón, pero estar muy satisfecho de la comodidad del mismo), no todos los clientes descontentos presentan quejas y reclamaciones. Por tanto, las empresas no conocen real y completamente la impresión global de sus clientes, ya que suponen que el cliente que no reclama está conforme con el producto o servicio recibido.

\section{b) Deficiencias relativas a la satisfacción de necesidades}

Algunas de las deficiencias observadas, tienen que ver con comportamientos inadecuados en una serie de procesos y tareas relacionados directamente con la satisfacción de esas necesidades, preferencias y requisitos que presentan los clientes de la organización. Varias de ellas se relacionan con actuaciones de planificación y tienen lugar en las instalaciones de la organización. El resto están relacionadas con el proceso de comercialización de los productos elaborados por la organización y el trato otorgado a sus clientes.

- Necesidad de una mayor formalización en la comunicación entre las diferentes áreas y secciones de la organización implicadas en el proceso productivo y la gestión de la calidad. En numerosas empresas hemos detectado una escasa coordinación entre los departamentos de diseño y de producción, y de éstos con el comercial a la hora de diseñar y producir los productos que los comerciales deberán de vender para satisfacer las necesidades del mercado. Esta falta de comunicación formalizada entre partes es contraria a las indicaciones de la norma ISO 9001, que en su epígrafe 8.2.4 plantea la necesidad de "hacer un seguimiento de las características del producto para verificar que se cumplen los requisitos del mismo" y que en reiterados epígrafes insiste en la necesidad del desarrollo de actuaciones coordinadas y documentadas, como es 
el caso del epígrafe 7.3.4, cuando especifica que en las revisiones sistemáticas del diseño y desarrollo deben participar "representantes de las funciones relacionadas con la (s) etapa(s) de diseño y desarrollo que se está(n) revisando. Deben mantenerse registros de los resultados de las revisiones y de cualquier acción necesaria".

- Falta de concienciación de gran parte de los operarios respecto de la trascendencia de su tarea individual en el éxito o fracaso comercial de los productos de la empresa. Este aspecto es muy relevante, y la norma ISO 9001, en su epígrafe 6.2.2, manifiesta que la organización debe "asegurarse de que su personal es consciente de la pertinencia e importancia de sus actividades y de cómo contribuyen al logro de los objetivos de la calidad". También la norma ISO 9004, en su epígrafe 6.2.2.2 así lo establece, cuando mantiene que la educación y la formación del personal "deberían enfatizar la importancia del cumplimiento de los requisitos y las necesidades y expectativas del cliente (...)". Si cada uno de los miembros de la organización no es consciente de que con su actuación personal e individualizada es responsable de que se satisfagan o no las necesidades y expectativas de los clientes, es difícil que el cliente pueda quedar satisfecho ${ }^{4}$. El compromiso de esa concienciación recae en la Dirección, quien debe comunicar a la organización "la importancia de satisfacer tanto los requisitos del cliente como los legales y reglamentarios" (apartado 5.1. Compromiso de la Dirección) y quien debe designar a un miembro responsable y con autoridad que promueva "la toma de conciencia de los requisitos del cliente en todos los niveles de la organización" (apartado 5.5.2. Responsabilidad, autoridad y comunicación).

- Prácticamente todos los casos analizados carecen de una planificación que fije objetivos comerciales para cada uno de los ejercicios económicos. La planificación comercial, que se configura como un instrumento que propone metas comerciales a conseguir en un periodo determinado, ajustadas a la realidad del mercado y a las posibilidades reales de la organización, no sólo racionaliza la actividad comercial de la empresa, sino que permite una mayor satisfacción de sus clientes y un proceso de mejora en el desempeño de la organización, pues la no consecución de los objetivos implica un análisis de las causas que explican esa circunstancia. Todos estos efectos derivados de la planificación comercial, son coherentes con la norma ISO 9001, pues ésta tiene como uno de sus objetivos la mejora continua de la organización, mediante acciones correctivas y de prevención, entre otras, que difícilmente podrían llevarse a cabo sin una planificación como la que consideramos. Y de hecho, en la norma ISO 9004 se considera como un referente básico, indicando en su epígrafe 5.1.1 a que "la alta dirección debería definir también métodos para medir el desempeño de la organización con el fin de determinar si se han alcanzado los objetivos planificados".

- Prevalencia de criterios de coste en lugar de otros relacionados con la calidad, para seleccionar tanto a proveedores como a transportistas, a pesar de la gran importancia que ambos tienen en la generación de satisfacción o insatisfacción de los clientes ${ }^{5}$. La propia norma 9001, en que apartado 6.1., estable que "la organización debe deter-

\footnotetext{
${ }^{4}$ La mayoría de las empresas comunican al resto de la organización la importancia de satisfacer las necesidades de los clientes de modo verbal. Tan sólo algunas certificadas utilizan otros medios como póster o boletines informativos.

${ }^{5}$ Según las empresas estudiadas la mayoría de reclamaciones efectuadas por los clientes están relacionadas con incidencias producidas durante el transporte de los productos.
} 
minar y proporcionar los recursos necesarios para (...) aumentar la satisfacción del cliente mediante el cumplimiento de sus requisitos". Además en su epígrafe, 7.5.5, por ejemplo, obliga a la organización a preservar la conformidad del producto durante el proceso de entrega al destino previsto, y el epígrafe 7.5, en general, establece que "la organización debe planificar y llevar a cabo la producción y la prestación del servicio bajo condiciones controladas". El cumplimiento de estas disposiciones, sin duda, reduciría las quejas de clientes que las empresas de nuestro estudio reciben por aspectos relacionados con el servicio de transporte e instalación que prestan a sus clientes.

- Falta de documentación adecuada en la tramitación de las reclamaciones a los clientes. La norma ISO 9001, en su epígrafe 8.2.1, enfatiza la importancia que tiene el seguimiento de la satisfacción del cliente, "como una de las medidas del desempeño del sistema de gestión de la calidad”; por lo tanto, la documentación de las reclamaciones de los clientes que permita una evaluación global de las mismas podría ser un instrumento de medición de dicha satisfacción. Sin embargo, en la mayoría de las empresas analizadas en la investigación de campo, la tramitación de las reclamaciones de los clientes se limita a la atención y resolución puntual de cada una de las quejas individuales recibidas, y muy pocas de estas empresas evalúan con posterioridad y de forma sistemática y documental el conjunto de quejas recibidas para detectar posibles orígenes de insatisfacción en los clientes. Evidentemente, esta falta de análisis global y sistemático impide, en muchos casos, que la organización se plantee acciones preventivas eficaces en función de las quejas recibidas, incumpliendo de esta forma lo dispuesto por la norma ISO 9001, que en su epígrafe 8.5.3 establece que "la organización debe determinar acciones para eliminar las causas de no conformidades potenciales para prevenir su ocurrencia”.

\section{CONCLUSIONES, RECOMENDACIONES Y FUTURAS ACTUACIONES}

Como se ha podido observar en los resultados mostrados, el cliente juega un papel significativo dentro de este sistema de gestión de la calidad, donde es preceptivo detectar sus necesidades con el fin de satisfacerlas. De este modo la organización debe adoptar un "enfoque al cliente" donde la Dirección tome un papel esencial en el proceso de concienciación y comunicación, a todos los niveles de la organización, de la importancia de satisfacer los requerimientos del cliente. A continuación, se plantean una serie de recomendaciones, que podrían corregir razonablemente las deficiencias anteriormente destacadas, y ayudarían a cumplir los requisitos de la norma de forma más eficiente (ver Tabla 2).

- Arbitrar sistemas que fortalezcan la lealtad de los comisionistas de las diferentes empresas. Si no es posible aumentar dicha lealtad, mediante el incremento de las comisiones contratadas, habría que intentarlo mediante planes que vinculen más al comisionista con la empresa y sus productos. Esta mayor lealtad del comisionista, podría conseguirse -en caso de no poder mejorar sus retribuciones económicas- desarrollando actuaciones que generen sinergias entre comisionistas y empresa, como aportándoles formación o apoyo promocional si lo necesitaran, concediéndoles cierta exclusividad ligada al cumplimiento de objetivos, facilitándoles los procesos relacionados con el cobro de sus comisiones, e integrándolos en mayor medida en la co- 
munidad de intereses y relaciones que forma la empresa. De esta forma se lograrían, entre otros, los siguientes efectos: (1) tener una fuerza de ventas más fiable y más estable; (2) aumentar la facturación; y (3) mejorar la calidad de las comunicaciones entre empresa y mercado.

- Facilitar la comunicación directa de la empresa con sus clientes. Esto podría conseguirse diseñando un plan de visitas periódicas a los principales clientes, realizando mailings para conocer las opiniones y satisfacción de los clientes y usuarios finales, o mediante la realización de una página web en la que se facilitara completa información de la empresa y sus productos, y a través de la cual se permitiera el envío directo de reclamaciones, quejas, o comentarios por parte de los clientes, así como de los distribuidores, estableciéndose como un cauce de comunicación directa de bastante utilidad. Con respecto a los mailings, se podría incentivar su contestación, generalmente escasa, mediante algún pequeño obsequio o sorteo entre quienes los respondiesen. En cuanto a la página $w e b$, además de habilitarla como medio de recepción de quejas y reclamaciones, podría utilizarse también como un vehículo apropiado para hacer llegar a los clientes un cuestionario mediante el que se pudiera evaluar las opiniones y satisfacción de los clientes y usuarios, y que al realizarse por Internet tendría una tasa de respuesta mayor, entre los clientes internautas, que la que se obtiene generalmente como respuesta de los cuestionarios enviados a través de mailings más convencionales. Así, se alcanzarían, entre otros, los siguientes efectos: (1) mejora de la imagen de la empresa entre clientes y usuarios; (2) mejor conocimiento de la satisfacción de clientes y usuarios, así como de sus necesidades, preferencias y gustos; y (3) menor dependencia de los comisionistas, que ofrecen una opinión sesgada sobre clientes y usuarios. Establecer mecanismos de sistematización de la información que se recoge acerca de tendencias, modas, preferencias de los mercados. Esta revisión y análisis de la información debería de hacerse de forma periódica, y de forma colegiada entre personas que, pertenecientes a distintas áreas de la empresa, pudieran aportar sus propios puntos de vista. En consecuencia, se podrían conseguir, los siguientes efectos: (1) que no se analicen las preferencias y tendencias del mercado sólo de forma ocasional o coyuntural, y en base a datos pasados o presentes (no de anticipación); (2) reducir el subjetivismo a la hora de interpretar posibles tendencias; y (3) que las decisiones sobre compras y nuevos productos no excluyan a áreas tan significativas como la comercial.

- Mejorar la comunicación interna con los miembros de la organización. Esto podría conseguirse mediante reuniones periódicas entre las distintas secciones y áreas implicadas. De esta forma, se lograría: (1) tomar las decisiones desde un espectro de puntos de vista e interés mucho más amplio que el habitual; (2) mayor consenso entre las diferentes áreas de gestión de la empresa; y (3) mayor integración de las diferentes áreas en un proyecto común.

- Introducir el concepto de cliente interno dentro de la estructura organizativa de la propia empresa. Esto podría conseguirse mediante la adecuada concienciación de los empleados implicados, vía motivación, o a través de la implantación de un sistema de parte de incidencias sección a sección, operario a operario. Así, se conseguirían los siguientes efectos: (1) concienciar a todos los componentes de la organización, de la necesidad de responder de la calidad objetiva de su trabajo; (2) reducir el número de incidencias 
y disconformidades en el proceso de producción de los diferentes bienes; (3) mayor motivación de los empleados al sentirse cada uno un elemento importante del proceso.

- Implantación de un análisis detallado de las reclamaciones de los clientes, tanto a nivel de distribuidor, como a nivel de usuario final, para poder detectar posibles aspectos de mejora. Esto podría conseguirse si las reclamaciones se tramitaran de forma adecuada y estandarizada, se archivaran debidamente, y periódicamente se analizaran tanto de forma global como individualizada. De este modo, se podría conseguir: (1) una reducción significativa del promedio de tiempo de atención al cliente reclamante; (2) mejora de la calidad al detectar posibles fallos en el proceso productivo y desempeños mejorables; y (3) posibilidad de utilizar las quejas, reclamaciones y sugerencias de los clientes para poder generar nuevos productos o modificaciones sustanciales en los presentes.

- Diseño de un proceso constante y sistemático de análisis de la competencia. Esto podría obtenerse mediante la recogida formalizada de informaciones en ferias, tiendas y comisionistas, no limitándose sólo a la recogida de datos sobre precios y estilos, sino también a informaciones sobre estrategias comerciales, mercados, proveedores, etc. Esta acción conllevaría diversos efectos : (1) no limitarse a un análisis ocasional o coyuntural por una posible falta de ideas; (2) una importante información para la dirección estratégica de la empresa; y (3) aumentar el número de ideas sobre nuevos productos, analizando lo que la competencia hace o no hace.

- Instaurar un sistema de clasificación de proveedores y contratas para asegurar que a la empresa que los productos comprados o la actividad solicitada cumplen con los requisitos establecidos. Para ello la empresa puede elaborar un ranking evaluando a las empresas contratas en base a una serie de criterios como: antigüedad, puntualidad, calidad suministros, lealtad, compromiso, seguridad, flexibilidad, etc. La implantación de dicho sistema de evaluación por parte de la empresa podría derivar en: (1) mayor seguridad de que los suministros se ajustan a los requerimientos exigidos; (2) control en el proceso logístico; (3) puntualidad en las entregas; (4) reducción de quejas por parte de los usuarios finales.

- Establecer acciones de testado de prototipos con los principales clientes de la empresa. Esto podría realizarse en las ferias, o diseñando un plan que facilite e incentive las visitas de los principales clientes a la empresa. De este modo, se alcanzarían los siguientes resultados: (1) reducir la incertidumbre implícita en el proceso de creación y desarrollo de nuevos productos; (2) lograr una mayor adaptación de los productos nuevos a las preferencias y necesidades reales de los principales clientes; y (3) facilitar la necesaria comunicación directa y la integración entre la empresa y sus principales clientes.

- Fijar objetivos comerciales razonables, para encauzar todos los esfuerzos de la organización al logro de dichos objetivos. Estos objetivos comerciales deberían de fijarse en función de los resultados obtenidos en un análisis previo de la realidad interna (recursos, capacidades y limitaciones) de la empresa y de la realidad externa (mercado, entorno, competencia), de forma que sean objetivos realistas y, al mismo tiempo, coherentes con los objetivos generales de la empresa y sus posibilidades reales. Para favorecer su posterior control, estos objetivos comerciales se formularán de forma cuantificable, utilizando variables medibles, y su cumplimiento estará referido a un 
plazo de tiempo determinado. Una vez pasado ese lapso temporal, será el momento de comprobar si los objetivos comerciales fijados se han logrado o no. En caso de que no se logren dichos objetivos, habría que analizar los motivos de esos incumplimientos y tomar medidas para que los objetivos puedan ser alcanzados en futuros ejercicios. De esta forma, se lograría: (1) relacionar de un modo más evidente los recursos, las tareas y los logros obtenidos; (2) facilitar la detección de posibles mejoras en el desempeño comercial, o de otro tipo, de la empresa; y (3) que la empresa determine las metas y logros que desea conseguir.

Tabla 2

Resumen de las deficiencias encontradas y las recomendaciones propuestas y sus efectos

\begin{tabular}{|c|c|c|}
\hline Deficiencias & Recomendaciones & Efectos \\
\hline $\begin{array}{l}\text { Falta de un proceso } \\
\text { formal de análisis de } \\
\text { tendencias y preferen- } \\
\text { cias del mercado }\end{array}$ & $\begin{array}{l}\text { Establecer mecanismos de que } \\
\text { recojan las tendencias, modas, } \\
\text { preferencias de los mercados. }\end{array}$ & $\begin{array}{l}\text { (1) Análisis sistemático; (2) reduc- } \\
\text { ción del subjetivismo; y (3) inclusión } \\
\text { área comercial en compras y nuevos } \\
\text { productos }\end{array}$ \\
\hline $\begin{array}{l}\text { Falta de un proceso } \\
\text { formal y estandarizado } \\
\text { de análisis de la com- } \\
\text { petencia }\end{array}$ & $\begin{array}{l}\text { Proceso de análisis constante, } \\
\text { sistemático y detallado de la } \\
\text { competencia a través de ferias, } \\
\text { tiendas y comisionistas. }\end{array}$ & $\begin{array}{l}\text { (1) Evitar análisis ocasionales por } \\
\text { falta de ideas; ( } 2 \text { ) disponer de infor- } \\
\text { mación para la dirección estratégica; } \\
\text { y (3) desarrollo nuevos productos } \\
\text { basándose en la competencia. }\end{array}$ \\
\hline $\begin{array}{l}\text { Ausencia de investi- } \\
\text { gación de mercados } \\
\text { para testar nuevos } \\
\text { productos }\end{array}$ & $\begin{array}{l}\text { Establecer acciones de testado } \\
\text { de prototipo a través de las } \\
\text { visitas de los principales clientes } \\
\text { a la empresa o en ferias. }\end{array}$ & $\begin{array}{l}\text { (1) Reducir la incertidumbre en el } \\
\text { desarrollo de nuevos productos; (2) } \\
\text { mayor adaptación de las novedades a } \\
\text { las preferencias y necesidades de los } \\
\text { clientes; y (3) facilitar la comunica- } \\
\text { ción e integración de la empresa con } \\
\text { sus clientes. }\end{array}$ \\
\hline $\begin{array}{l}\text { Falta de comunicación } \\
\text { directa de las empresas } \\
\text { con sus clientes, y con } \\
\text { los usuarios finales }\end{array}$ & $\begin{array}{l}\text { Con clientes: Arbitrar sistemas } \\
\text { que fortalezcan la lealtad de los } \\
\text { comisionistas. Plan de visitas } \\
\text { periódicas a los principales } \\
\text { clientes. } \\
\text { Con usuarios finales: Mailing } \\
\text { o web como medios directos } \\
\text { de reclamaciones, quejas, o } \\
\text { comentarios. }\end{array}$ & $\begin{array}{l}\text { (1) Tener una fuerza de ventas más } \\
\text { fiable y más estable; (2) aumentar la } \\
\text { facturación; ( } 3 \text { ) mejorar la comuni- } \\
\text { cación entre empresa y mercado; (4) } \\
\text { mejora de la imagen de la empresa } \\
\text { entre clientes y usuarios; (5) mejor } \\
\text { conocimiento de las preferencias y } \\
\text { satisfacción de clientes y usuarios; (6) } \\
\text { menor dependencia de los comisionis- } \\
\text { tas que ofrecen una opinión sesgada. }\end{array}$ \\
\hline $\begin{array}{l}\text { Necesidad de una ma- } \\
\text { yor formalización en la } \\
\text { comunicación entre las } \\
\text { áreas implicadas en el } \\
\text { proceso productivo y } \\
\text { la calidad. }\end{array}$ & $\begin{array}{l}\text { Reuniones periódicas entre } \\
\text { las distintas secciones y áreas } \\
\text { implicadas. }\end{array}$ & $\begin{array}{l}\text { (1) Tomar las decisiones desde puntos } \\
\text { de vista más amplios; (2) mayor } \\
\text { consenso entre las áreas; y ( } 3 \text { ) mayor } \\
\text { integración de las áreas en un proyec- } \\
\text { to común. }\end{array}$ \\
\hline
\end{tabular}




\begin{tabular}{|c|c|c|}
\hline Deficiencias & Recomendaciones & Efectos \\
\hline $\begin{array}{l}\text { Falta de conciencia- } \\
\text { ción de gran parte de } \\
\text { los operarios respecto } \\
\text { de la trascendencia } \\
\text { de su tarea individual } \\
\text { en el éxito o fracaso } \\
\text { comercial de los pro- } \\
\text { ductos de la empresa }\end{array}$ & $\begin{array}{l}\text { Introducir el concepto de cliente } \\
\text { interno dentro de la estructura } \\
\text { organizativa: motivando a em- } \\
\text { pleados, implantando partes de } \\
\text { incidencias sección a sección, } \\
\text { operario a operario. }\end{array}$ & $\begin{array}{l}\text { (1) Concienciar a toda la organización } \\
\text { de la calidad objetiva de su trabajo; } \\
\text { (2) reducir las incidencias y discon- } \\
\text { formidades en la producción; (3) } \\
\text { mayor motivación del empleado al } \\
\text { sentirse parte del proceso. }\end{array}$ \\
\hline $\begin{array}{l}\text { Carencia de planifi- } \\
\text { cación de objetivos } \\
\text { comerciales }\end{array}$ & $\begin{array}{l}\text { Tras un análisis interno y exter- } \\
\text { no fijar objetivos comerciales } \\
\text { realistas, referidos a un tiempo } \\
\text { determinado, medibles y que } \\
\text { sean coherentes con otros objeti- } \\
\text { vos de la empresa. Controlar su } \\
\text { cumplimiento. }\end{array}$ & $\begin{array}{l}\text { 1) Relacionar eficientemente los } \\
\text { recursos y tareas con los logros } \\
\text { obtenidos; (2) facilitar la detección } \\
\text { de posibles mejoras en el desempeño } \\
\text { comercial; y (3) determinar las metas } \\
\text { y logros que desea conseguir. }\end{array}$ \\
\hline $\begin{array}{l}\text { Prevalencia de crite- } \\
\text { rios de coste en lugar } \\
\text { de otros relacionados } \\
\text { con la calidad para se- } \\
\text { leccionar proveedores }\end{array}$ & $\begin{array}{l}\text { Elaborar un sistema de } \\
\text { clasificación de proveedores } \\
\text { atendiendo a criterios como: } \\
\text { antigüedad, puntualidad, calidad } \\
\text { suministros, lealtad, seguridad, } \\
\text { flexibilidad, etc. }\end{array}$ & $\begin{array}{l}\text { (1) Mayor seguridad en los suminis- } \\
\text { tros; } \\
\text { (2) control logístico; (3) puntualidad } \\
\text { en las entregas; (4) reducción de que- } \\
\text { jas por parte de los usuarios finales }\end{array}$ \\
\hline $\begin{array}{l}\text { Falta de documen- } \\
\text { tación adecuada en } \\
\text { la tramitación de las } \\
\text { reclamaciones a los } \\
\text { clientes }\end{array}$ & $\begin{array}{l}\text { Implantación de un sistema de } \\
\text { tramitación, archivo y análisis } \\
\text { periódico de las reclamaciones } \\
\text { de clientes y usuarios finales de } \\
\text { forma global y individualizada. }\end{array}$ & $\begin{array}{l}\text { 1) Reducir el tiempo tramitación } \\
\text { de quejas; (2) mejorar al detectar } \\
\text { posibles fallos en el proceso produc- } \\
\text { tivo y desempeños; y (3) utilizar las } \\
\text { reclamaciones y sugerencias para mo- } \\
\text { dificar o generar nuevos productos. }\end{array}$ \\
\hline
\end{tabular}

Estas recomendaciones ayudarían a las empresas del sector del mueble a desarrollar de forma más eficiente todos los postulados relacionados con la gestión del cliente establecidos en un sistema de calidad, como son las normas ISO 9000.

De análisis de los casos estudiados han emergido otras conclusiones dignas de ser recalcadas que nos ayudarán a entender donde se encuadra al cliente dentro de las empresas de este sector. Aunque aparentemente, el certificarse supone una cuestión de moda, una obligación en el mercado o una herramienta promocional, globalmente, podemos concluir que las empresas certificadas tienen una mayor concienciación por identificar y satisfacer las necesidades de consumidor, que las no certificadas. Además, la mayoría de las empresas certificadas han adoptado la norma más por motivos internos $(71,2 \%)$ que por motivaciones externas; es decir, se certifican además de por su propias exigencias por mejorar la calidad de sus bienes y/o servicios por los numerosos beneficios internos atribuidos a la certificación: beneficios organizativos y de control, de productividad y costes, y beneficios comerciales; ligados estos últimos a la mejora de la satisfacción, imagen e identificación de los requerimientos del cliente. Sin embargo, prácticamente la totalidad de las empresa analizadas reconocen que, si el resto de competidores están certificados, el hecho de no 
estarlo puede ser un agravio comparativo muy importe. De este modo, muestran que en su decisión de certificarse las exigencias externas propiciadas por la presión competitiva también son tenidas en cuenta.

Este estudio de casos, supone un primer paso en la investigación, nos ofrece un acercamiento al problema que podría ser complementado con el análisis empírico sobre este fenómeno. Aunque su replicación a otras empresas del sector es fácilmente realizable, dado el amplio número de casos analizados, la obtención de datos redundantes sería muy probable. Por ello, estudios posteriores deberían centrarse en corroborar las conclusiones obtenidas a través de un estudio empírico con mayor cobertura geográfica en el que se consulte a una muestra representativa de la industria del mueble española acerca de sus actuaciones, con el fin de determinar las necesidades del cliente y conseguir su satisfacción. De este modo, podremos elaborar un manual de las actuaciones más recomendables para alcanzar dichos propósitos en una industria tan especifica y competitiva como es la del mueble en España.

\section{REFERENCIAS}

AENOR (2001): Gestión de la Calidad. ISO 9000.2000. Madrid, AENOR.

ÁlVAREZ GIL, M. J., CORBETT, C., Y MONTES, M. J. (2002): "El impacto financiero de la certificación ISO 9000. Un análisis empírico", XII Congreso Nacional de ACEDE, Palma de Mallorca, España.

ANDERSON, E., FORNELL, C., Y LEHMANN, D. (1994): “Customer Satisfaction, Market share, and Profitability. Findings from Swede”, Journal of Marketing, Vol. 58, pp. 53-66.

ANDERSON, S. W., DALY, J. D., Y JOHNSON, M. F. (1999): “Why firms seek ISO 9000 certification. regulatory compliance or competitive advantage?", Production and Operations Management, Vol. 8, No. 1, pp. 28-43.

BENBASAT, I., GOLDSTEIN, D. K., Y MEAD, M. (1987): “The Case Research Strategy in Studies of Information Systems", MIS Quarterly, Vol. 11, No. 3, pp. 369-385.

BONOMA, T. V. (1985): "Case Research in Marketing. Opportunities, Problems, and a Process", Journal of Marketing Research, Vol. 22, pp. 199-208.

BROWN, A., VAN DER WIELE, T., Y LOUGHTON, K. (1998): “Smaller Enterprises' Experiences with ISO 9000", International Journal of Quality \& Reliability Management, Vol. 15, No. 3, pp. 273-285.

BROWN, A., Y VAN DER WIELE, T. (1995): "Industry experience with ISO 9000”, Asia Pacific Journal of Quality Management, Vol. 4, No. 2, pp. 8-17.

BUTTLE, F. (1997): "ISO 9000. Marketing Motivations and Benefits. International Journal of Quality \& Reliability Management, Vol. 14, No. 9, pp. 936-947.

CALISIR, F. (2007): "Factors affecting service companies' satisfaction with ISO 9000", Managing Service Quality, Vol. 17, No. 5, pp. 579-593.

CALISIR, F., KULAK, O., Y DOGAN, I. (2005): "Factors influencing Turkish textile companies' satisfaction with ISO 9000", Total Quality Management \& Business Excellence, Vol. 16, No. 10), pp. 1193-204.

CAMISÓN, C. (1996): "La Calidad como Factor de Competitividad en Turismo. Análisis del Caso Español”, Información Comercial Española, Vol. 749, No. Enero, pp. 99-122.

CARLSSON, M., Y CARLSSON, D. (1996): "Experiences of Implementing ISO 9000 in Swedish Industry", International Journal of Quality \& Reliability Management, Vol. 13, No. 7), pp. 36-47.

CARUANA, A., Y PITT, L. (1997): "INTQUAL-An Internal Measure for Service Quality and the Link between Service Quality and Business Performance", European Journal of Marketing, Vol. 31, No. 8, pp. 604-617. 
CASADESÚS, F. M., HERAS, I., Y ARANA, G. (2004): “Costes y Beneficios de la Implantación de la Normativa de Calidad ISO 9000. Evolución Temporal”, XIV Congreso Nacional de ACEDE, Murcia, España.

CASADESÚS, M., Y GIMÉNEZ, G. (2000): "The Benefits of the Implementation of the ISO 9000 Standard. Empirical Research in 288 Spanish Companies", The TQM Magazine, Vol. 12, No. 6, pp. 432-441.

CASADESÚS, M.; HERAS, I. Y ARANA, G. (2004): "Costes y beneficios de la implantación de las normativa de calidad ISO 9000. Evolución temporal", XIV Congreso Nacional de ACEDE, Murcia, España.

CHAPMAN, R., MURRAY, P., Y MELLOR, R. (1997): "Strategic quality management and financial performance indicators", International Journal of Quality \&Reliability Management, Vol. 14, No. 4, pp. 432-48.

CHOW-CHUA, C., GOH, M., Y BOON WAN, T. (2003): "Does ISO 9000 certification improve business performance?”, International Journal of Quality \& Reliability Management, Vol. 20, No. 8, pp. 936-53.

DIMARA, E., SAKURAS, D., TSEKOURAS, K., Y GOUTSOS, S. (2004): "Strategic orientation and financial performance of firms implementing ISO 9000", International Journal of Quality \& Reliability Management, Vol. 21, No. 1, pp. 72-89.

ESCANCIANO, C. (2001): "La Empresa Española y su Opinión sobre el ISO 9000. Análisis de los Resultados de un Estudio Empírico", Economía Industrial, 341, pp. 151-159.

ESCANCIANO, C., FERNÁNDEZ, E., Y VÁZQUEZ, C. (2001). "Influence of ISO 9000 Certification on the Progress of Spanish Industry towards TQM". International Journal of Quality and Reliability Management, Vol. 18, No. 5, pp. 481-494.

FERNÁNDEZ GONZÁLEZ, A. J. Y PRADO PRADO J. C. (2005): "Medición y análisis de la satisfacción del cliente. Prácticas en empresas de Galicia y Portugal”, IX Congreso de Ingeniería de Organización, Gijón, España.

FLYNN, B. B., SCHROEDER, R. G., FLYNN, E. J., SAKAKIBARA, S., Y BATES, K. A. (1997): "World-Class Manufacturing Project. Overview and Selected Results", International Journal of Operations and Production Management, Vol. 17, No. 7, pp. 671-685.

GOTZAMANI, K. D., Y TSIOTRAS, G. D. (2002): "The True Motives behind ISO 9000 Certification. Their Effect on the Overall Certification Benefits and their Long Term Contribution towards TQM", International Journal of Quality and Reliability Management, Vol. 19, No. 2, pp. 151-169.

GURAU, C., Y RANCHHOD, A. (2002): "How to Calculate the Value of a Customer", Journal of Targeting, Measurement and Analysis for Marketing, Vol. 10, No. 3, pp. 20-220.

HERAS, I., ARANA, G., CABEZUDO, S., Y CASADESÚS, M. (2005): "Los Modelos de Gestión de la Calidad y su Influencia sobre los Resultados de las Empresas", XV Congreso Nacional de $A C E D E$, Tenerife, España.

HERAS, I., OCHOA, C., Y ARANA, P. (2000): "Análisis Empírico de las Incidencias de la Normativa ISO 9000 en la Rentabilidad Económica de las Empresas", Revista de Economía y Empresa, Vol. 14, No. 39, pp. 29-44.

HERNÁNDEZ, R., MUÑOZ, P., SANTOS, L., Y GONZÁLEZ O. (2003): “Una propuesta general de análisis de la calidad y la satisfacción en el Turismo Rural Español”, XII Simposio Internacional de Turismo y Ocio, Barcelona, España.

JONES, R., ARNDT, G. Y KUSTIN, R. (1997): "ISO 9000 among Australian companies: impact of time and reasons for seeking certification on perceptions of benefits received", International Journal of Quality \& Reliability Management, Vol. 14, No. 7, pp. 650-660.

LEE, T. Y., LEUNG, H. K. N., Y CHAN, K. C. C. (1999): "Improving Quality Management on The Basis of ISO 9000", The TQM Magazine, Vol. 11, No. 2, pp. 88-94.

MAGD, H., Y CURRY, A. (2003a): "An empirical analysis of management attitudes towards ISO 9001:2000 in Egypt”, The TQM Magazine, Vol. 15, No. 6, pp. 381-90. 
MAGD, H., Y CURRY, A. (2003b): "ISO 9000 and TQM: are they complementary or contradictory to each other", The TQM Magazine, Vol. 15, No. 4, pp. 244-56.

MARIMON, F., CASADESÚS, M., Y HERAS, I. (2002): "Do Consultants Offer A Quality Service?", Total Quality Management Journal, Vol. 13, No. 6, pp. 797-811.

MARÍN VINUESA, L.M.; GIMENO ZUERA, J. Y RUIZ-OLALLA CORCHERA, M.C. (2007): "Influencia de los indicadores no financieros sobre los resultados de las empresas inmersas en procesos TQM. una evidencia empírica“. En Ayala J.C. (coord.), Conocimiento, innovación y emprendedores. camino al futuro (pp. 291-307). Logroño, Servicio de Publicaciones Universidad de la Rioja.

MARTÍNEZ, A. R., Y MARTÍNEZ, M. (2004): “ISO 9000 \& TQM. Substitutives or Complementaries? An Empirical Study in Industrial Companies", International Journal of Quality and Reliability Management, Vol. 21, No. 3, pp. 260-276.

MARTÍNEZ, J. A. Y MARTÍNEZ, L. (2009): "Does ISO 9000 Certification Affect Consumer Perceptions of the Service Provider?", Managing Service Quality, Vol. 19, No. 2, pp. 140-161.

MOHR-JACKSON, I. (1998): “Conceptualising Total Quality Orientation”, European Journal of Marketing, Vol. 32, No. 1/2, pp. 13-22.

PHILLIPS, L. W., CHANG, D. R., Y BUZZELL, R. D. (1983): "Product Quality, Cost Position, and Business Performance: A Test of Key Hypotheses", Journal of Marketing, Vol. 37, No. 1, pp. 26-43.

QUAZI, H. A., Y PADIBJO, S. R. (1997): "A journey towards total quality management through ISO 9000 certification - a Singapore experience", The TQM Magazine, Vol. 9, No. 5, pp. 364-71.

RODRÍGUEZ, J. A., Y GONZÁLEZ, J. (2003): "Un estudio de la Insatisfacción Generada por la Certificación ISO 9000 en la Pequeña Empresa”, XIII Congreso Nacional de ACEDE. Salamanca (España).

RUST, R. T., ZAHORIK, A. J., Y KEININGHAM, T. I. (1994): "Return on quality (ROQ): Making Service Quality Financially Accountable”, Journal of Marketing, Vol. 59, No. 2, pp. 58-70.

RUST, R.T., Y OLIVER, R. L. (1994): "Service Quality: Insights and Managerial Implications from the Frontier. En R. T. Rust, y R. L. Oliver (Eds.), Service Quality: New Directions in Theory and Practice (pp. 1-19). London, Sage Publications.

SANTOS, L., Y ESCANCIANO, C. (2000). "Análisis de la Influencia de la Certificación ISO 9000 sobre los Resultados Empresariales: Implicaciones en el ámbito de la Calidad Total y de la Orientación al Mercado". ICADE, Vol. 55, pp177- 204.

SASTRE, M. A. (2004): "El mueble. Cambio de estrategia“, Moneda única, Vol. 35. Recuperado de http.//www.monedaunica.net.

TSIOTRAS, G., Y GOTZAMANI, K. (1996): "ISO 9000 as an Entry Key to TQM. The Case of Greek Industry”, International Journal of Quality \& Reliability Management, Vol. 13, No. 4, pp. 64-76.

TERZIOVSKI, M., SAMSON, D., Y DOW, D. (1995). “The Impact of ISO 9000 Certification on Customer Satisfaction". Asia Pacific Journal of Quality Management, Vol. 4, No. 2,pp 66-68.

URBONAVICIUS, S. (2005): "ISO system implementation in small and medium companies from new EU member countries A tool of managerial and marketing benefits development", Research in International Business and Finance, Vol. 19, pp. 412-426.

YIN, R. (2003): Case study research. Design and methods (3“ Edición). Thousand Oaks, Sage.

YIN, R. K. (1994): Case Study Research, Design and Methods ( $2^{a}$ Edición). Beverly Hills, CA, Sage.

ZARAMDINI, W. (2007): "An empirical study of the motives and benefits of ISO 9000 certification: the UAE experience", International Journal of Quality \& Reliability Management, Vol. 24, No. 5, pp. 472-491. 